\title{
The Relationship Between Dry Eye Disease and Digital Screen Use
}

\author{
Zaina Al-Mohtaseb' \\ Scott Schachter ${ }^{2}$ \\ Bridgitte Shen Lee ${ }^{3}$ \\ Jaclyn Garlich (iD) \\ William Trattler ${ }^{5}$
}

'Department of Ophthalmology, Cullen Eye Institute, Baylor College of Medicine, Houston, TX, USA; ${ }^{2}$ Presbyopia and Ocular Surface Disease, Allergan, an AbbVie Company, Irvine, CA, USA; ${ }^{3}$ Vision Optique, Houston, TX, USA; ${ }^{4}$ Envision Optometry, Boston, MA, USA; ${ }^{5}$ Center for Excellence in Eye Care, Miami, FL, USA
Correspondence: Zaina Al-Mohtaseb Department of Ophthalmology, Cullen Eye Institute, Baylor College of Medicine, 6565 Fannin Street, Houston, TX, 77030, USA

Tel $+|-7| 3-798-5 \mid 43$

Fax +I-7|3-798-3027

Email zaina@bcm.edu

\begin{abstract}
Dry eye disease is characterized by tear film instability that can result in ocular surface damage. Patients with dry eye disease may experience ocular pain/discomfort and visual disturbances that may negatively impact quality of life. Increased use of digital screens for work, communication, and entertainment, especially during times of pandemic, may contribute to dry eye. Extensive cross-sectional studies have shown that digital screen use duration is associated with an increased risk of severe symptoms and clinical diagnosis of dry eye disease in adults. Smartphone use duration has also been found to be greater in school-age children with dry eye disease than in those without dry eye disease. A commonly accepted hypothesis for the relationship between digital screen use and dry eye disease is that digital screen use changes blinking dynamics, leading to ocular dryness. This review describes evidence that digital screen use is associated with dry eye disease, that digital device use alters blinking dynamics, and that dry eye affects mental health and work productivity in digital screen users. Helpful prevention and management strategies for dry eye disease exist for those who use digital screens.
\end{abstract}

Keywords: blinking, ocular surface, quality of life, smartphone, computer, visual display

\section{Introduction}

Dry eye disease (DED) is a disease of the ocular surface, characterized by tear film instability and inflammation, which can potentially damage the ocular surface. ${ }^{1}$ Estimates of the prevalence of dry eye in the population range widely from $5 \%$ to $50 \%$, which is likely due in part to varying definitions of DED. ${ }^{2}$ Symptoms of dry eye vary in severity between individuals and can include ocular discomfort, pain, fatigue, and visual disturbances, such as fluctuating and blurry vision. ${ }^{1,3,4}$ The discomfort and pain from dry eye is thought to have a negative impact on quality of life and may affect mental health. ${ }^{2,5,6}$ Visual disturbances and discomfort may interfere with activities such as reading and driving. ${ }^{2,7,8}$ Furthermore, dry eye may affect work productivity, which has ramifications for personal success and the economy. ${ }^{9,10}$

Given the potentially negative impact of dry eye, it is important to understand the factors that contribute to its development. The etiology of dry eye can be difficult to determine because dry eye can be related to a number of different factors. Commonly accepted risk factors for DED include intrinsic factors such as increasing age, female gender, ocular diseases, and certain underlying systemic and autoimmune diseases. ${ }^{2}$ Extrinsic risk factors for DED may include contact lens wear, environmental conditions (eg, low humidity or airflow on the eye), topical or systemic medications, lack of hygienic practices for eyelids and eyelashes, eye beauty trends, and eye cosmetic product ingredients and applications. ${ }^{2,11,12}$ 
One increasingly common extrinsic risk factor for dry eye is digital screen use (eg, computer, laptop, tablet, and smartphone use), which is thought to contribute to its development by affecting blinking dynamics. ${ }^{2,13}$ According to a metaanalysis, the estimated prevalence of DED in workers who use digital screens ranged from $9.5 \%$ to $87.5 \% .{ }^{14}$ Digital screen use is particularly relevant in the present era because of the common use of personal computers and smartphones. ${ }^{13,15,16}$ As an example, a recent study of 9- to 10year-old children $(\mathrm{N}=11,875)$ found that children used screens (for TV, video, video-game playing, texting, chatting, and social media) for an average of 3.8 hours per day and almost all children reported using screens daily. ${ }^{17}$ The COVID-19 pandemic increased the extent to which individuals use digital screens because of the need to stay at home and the incentive to work, learn, and socialize remotely. ${ }^{16,18-22}$ For many individuals, in-person school and meetings were closed or cancelled and replaced with virtual learning and events. ${ }^{16,23-25} \mathrm{In}$ a large survey in France during containment for the pandemic, $64 \%$ of respondents $(\mathrm{N}=11,391)$ reported an increase in screen use. ${ }^{26}$ Similarly, over $60 \%$ of respondents to a survey in Canada ( $\mathrm{N}=4524)$ reported increased Internet use during the pandemic, and $24 \%$ of men and $16 \%$ of women reported increased video-game use. ${ }^{27}$ In a survey of 4- to 17-year-olds in Germany $(\mathrm{N}=1717)$, total recreational screen time (TV watching, Internet usage, and gaming) was reported to increase by 61.2 minutes per day. ${ }^{21}$ This increase in screen use may lead to increased dry eye symptoms. ${ }^{18,28}$ In this review, we focus on the relationship between dry eye and digital screen use, describe factors that may account for the relationship, and discuss helpful prevention and management strategies for dry eye in those who use digital screens.

\section{Methods}

A MEDLINE/PubMed database search was conducted to review the literature on the topic of DED as it relates to digital screen use. We included articles from all years. Key search terms used were "dry eye," "visual display," "blink," "digital screen," and "screen use" in various combinations. Articles were reviewed and included for a narrative review if the information was pertinent to discussing the association between dry eye and digital screen use, the association between dry eye and blinking dynamics, the impact of dry eye on quality of life of digital screen users, or preventative strategy for dry eye in digital screen users. References within articles were also reviewed for inclusion.

\section{Relationship Between Digital Screen Use and Dry Eye Disease}

Several large cross-sectional studies have demonstrated a relationship between digital screen use and dry eye (Table 1). In a large study of office workers $(\mathrm{N}=3549)$, severe symptoms of dry eye were more prevalent among those who used digital screens for $>4$ hours per day (odds ratio $[\mathrm{OR}]=1.83) .{ }^{29}$ However, no significant relationship was found between duration of screen work and clinically diagnosed DED in this study. ${ }^{29}$ Notably, the position of the screen relative to the individual's eyes and use of a glare filter on the screen were not found to affect the risk of severe symptoms of dry eye or clinically diagnosed DED. ${ }^{29}$ Similarly, a large study using crowdsourcing data $(\mathrm{N}=4454)$ found an association between $>8$ hours of screen use per day and symptomatic dry eye (Ocular Surface Disease Index [OSDI] total score $\geq 13$ ) compared to $<4$ hours per day. ${ }^{30}$ Evidence also supports a relationship between duration of digital screen use and diagnosed DED. ${ }^{31-34}$ The OSAKA study $(\mathrm{N}=561)$ demonstrated that office workers who used digital screens for $>8$ hours per day had a higher risk of definite or probable DED $(\mathrm{OR}=1.94) .{ }^{31}$ Furthermore, the JPHC-NEXT study $(\mathrm{N}=102,582)$ found that greater digital screen use was associated with a higher risk of clinically diagnosed $\operatorname{DED}(\mathrm{OR}=1.18$ for each 1 hour/day increment) and severe symptoms of dry eye $(\mathrm{OR}=1.11$ for men and $\mathrm{OR}=1.12$ for women for each 1 hour/day increment). ${ }^{32}$ When risk factors for aqueous deficient DED and evaporative DED were examined among a group of 1125 individuals, greater digital screen exposure was found to be a predictor of evaporative DED. ${ }^{34}$ Although a relationship between screen use and diagnosed DED has been found, this relationship was not found when only individuals who had symptomatic dry eye were examined (OSDI total score $>13 ; \mathrm{n}=2395){ }^{30}$ Overall, these findings established a relationship between DED and digital screen use.

The association between digital screen use and DED has also been found in school-age children, specifically smartphone use. Among a group of 288 children in Korea (age range $=10-12$ years), the prevalence of smartphone use was higher among children with DED (71.4\%) than among children without DED (50\%). ${ }^{35}$ Furthermore, the daily duration of smartphone use $(\mathrm{OR}=1.86)$ and total daily duration of digital screen use $(\mathrm{OR}=1.82)$ were associated with an increased risk of DED. In contrast, daily duration of computer or television use was not found to be associated 
Table I Studies Investigating the Relationship Between Digital Screen Use and Symptoms of Dry Eye or Dry Eye Disease

\begin{tabular}{|c|c|c|}
\hline Reference & Sample & Finding \\
\hline Hikichi et al, $1995^{77}$ & $\begin{array}{l}\text { New outpatients at eye centers } \\
(\mathrm{N}=2127 \text {; age range }=10-92 \text { years })\end{array}$ & $\begin{array}{l}\text { I33 (6\%) individuals used digital screens. The prevalence of DED was higher } \\
\text { among those who used digital screens }(30 / / 33 ; 23 \%) \text {. }\end{array}$ \\
\hline Uchino et al, $2008^{29}$ & Office workers $(\mathrm{N}=3549)$ & $\begin{array}{l}\text { Severe symptoms of dry eye were more prevalent among those who used digital } \\
\text { screens for }>4 \text { hours per day }(O R=1.83) \text {. }\end{array}$ \\
\hline Uchino et al, $2013^{31}$ & Office workers $(\mathrm{N}=56 \mathrm{I})$ & $\begin{array}{l}\text { Those who used digital screens for }>8 \text { hours per day had a higher risk of definite } \\
\text { or probable DED }(O R=1.94) \text {. }\end{array}$ \\
\hline Moon et al, $2014^{35}$ & $\begin{array}{l}\text { Children }(\mathrm{N}=288 \text {; age range }=10-12 \\
\text { years) }\end{array}$ & $\begin{array}{l}\text { Prevalence of smartphone use was higher among children with DED }(71.4 \% \text { vs } \\
50 \%) \text {. Daily duration of smartphone use }(O R=I .86) \text { and total daily duration of } \\
\text { digital screen use }(O R=1.82) \text { were associated with an increased risk of DED. }\end{array}$ \\
\hline $\begin{array}{l}\text { Kawashima et al, } \\
2015^{4}\end{array}$ & Office workers $(\mathrm{N}=369)$ & $\begin{array}{l}\text { Duration of digital screen use was longer in those with DED ( } 6.5 \text { hours vs } 6.0 \\
\text { hours). }\end{array}$ \\
\hline Moon et al, $2016^{36}$ & Children $(\mathrm{N}=916$; age range $=7-12)$ & $\begin{array}{l}\text { Prevalence of smartphone use was higher in the DED group than the non-DED } \\
\text { group ( } 96.7 \% \text { vs } 55.4 \%) \text {. Daily duration of smartphone and computer use were } \\
\text { higher in the DED group ( } 3.18 \text { hours and I. } 10 \text { hours) than in the non-DED group } \\
\text { ( } 0.62 \text { hours and } 0.76 \text { hours). }\end{array}$ \\
\hline Hanyuda et al $2020^{32}$ & Adults $(\mathrm{N}=102,582)$ & $\begin{array}{l}\text { Greater digital screen use was associated with a higher risk of clinically diagnosed } \\
D E D(O R=I .18 \text { for men and } O R=I .18 \text { for women for each } I \text { hour } / \text { day increment) } \\
\text { and severe symptoms of dry eye (OR=I.II for men and } O R=I .12 \text { for women for } \\
\text { each } I \mathrm{~h} / \text { day increment). }\end{array}$ \\
\hline Inomata et al $2020^{30}$ & Adults $(\mathrm{N}=4454)$ & $\begin{array}{l}\text { Greater than } 8 \text { hours per day of screen exposure was associated with } \\
\text { symptomatic dry eye (OSDI total score } \geq \mid 3 ; O R=I .55) \text { compared to less than } 4 \\
\text { hours. }\end{array}$ \\
\hline Wang et al $2021^{33}$ & Individuals $\geq 16$ years of age $(\mathrm{N}=322)$ & Greater digital screen time per day was a risk factor for DED (OR=I.I4). \\
\hline Wolffsohn et al $2021^{34}$ & Adults and children $(\mathrm{N}=1 \mid 25)$ & $\begin{array}{l}\text { Digital screen time per day was a risk factor for DED }(O R=1.09) \text { and for } \\
\text { evaporative DED }(O R=1.08) \text {. }\end{array}$ \\
\hline
\end{tabular}

Abbreviations: DED, dry eye disease; OR, odds ratio; OSDI, Ocular Surface Disease Index.

with DED. ${ }^{35}$ These findings were confirmed in a larger study of children in Korea ( $\mathrm{N}=916$; age range=7-12). In this study, the prevalence of smartphone use was higher in children with DED (96.7\%) than in children without DED $(55.4 \%) .{ }^{36}$ In addition, the mean daily duration of smartphone and computer use was higher in the DED group (3.18 hours and 1.10 hours, respectively) than in the non-DED group (0.62 hours and 0.76 hours, respectively) ${ }^{36}$ In a multivariate analysis, the duration of smartphone use (but not the duration of computer or television use) was predictive of DED. ${ }^{36}$ When the children with DED ceased smartphone use for four weeks, all children showed improvement of DED as measured by superficial punctate epithelial erosions, tear breakup time, and OSDI score, which suggests lifestyle modifications can help to improve DED in some young digital screen users. ${ }^{36}$

\section{Relationship Between Digital Screen Use and Ocular Surface Measures}

Several studies have assessed the relationship between digital screen use and ocular surface metrics, including tear breakup time, tear volume (eg, tear meniscus height and Schirmer score), and tear film lipid layer status.

A small study by Cardona et al found several changes in the tear film after 20 minutes of video-game playing on a computer screen in 25 healthy young adults. ${ }^{37}$ Tear meniscus height decreased, tear breakup time decreased, the tear breakup area increased, and the interference patterns of the lipid layer changed following video-game playing. ${ }^{37}$ A later study examined changes in tear film over the course of a workday (ie, at 8 am and $5 \mathrm{pm}$ ) in a group who worked approximately 8 hours per day on 
computers $(n=30)$ and a group who worked $<1$ hour per day on display screens $(n=30) .{ }^{38}$ No significant change was observed in Schirmer score (with topical anesthesia) over the workday for either group, but tear breakup time decreased from 9.15 seconds in the morning to $6.80 \mathrm{sec}-$ onds in the evening in the digital screen workers. ${ }^{38}$ In the group that worked $<1$ hour per day on digital screens, tear breakup time was approximately 15 seconds in the morning and the evening. ${ }^{38}$ When different digital devices have been compared, reading on a computer resulted in lower tear meniscus height, higher osmolarity, and greater conjunctival redness compared to reading on a smartphone, possibly because reading on a smartphone was associated with a lower gaze angle and lower extent of exposed ocular surface. ${ }^{39}$ The results of these studies suggest digital screen use is associated with an acute deterioration of tear film quality.

There is also some evidence suggesting that years of digital screen use is associated with a reduced aqueous component of the tear film. In a study of 1025 office workers, $>8$ hours of daily digital screen use was associated with a reduced Schirmer I score $(\leq 5 \mathrm{~mm})$ compared with $<2$ hours of daily digital screen use $(\mathrm{OR}=4.27) .{ }^{40}$ Furthermore, working on digital screens for $8-12$ years $(\mathrm{OR}=2.49)$ or $>12$ years $(\mathrm{OR}=3.61)$ was associated with a reduced Schirmer I score ( $\leq 5 \mathrm{~mm}$ ) compared to $<4$ years, suggesting an effect of cumulative years of digital screen use. However, no relationship was found between digital screen use and tear film breakup time or tear film lipid status. ${ }^{40}$ A smaller study of 69 contact lens wearers and 102 non-contact lens wearers also found evidence that digital screen use affects the aqueous component of the tear film. ${ }^{41}$ Mean tear meniscus height was lower in those who used digital screens for $\geq 4$ hours per day; however, no significant association was found between digital screen use and Schirmer I score. In addition, tear film breakup time and ocular surface staining showed no association with digital screen use. ${ }^{41}$ In contrast, the OSAKA study found most office workers had short tear breakup time and normal Schirmer score, but the specific association between digital screen use and clinical measures of dry eye was not examined. ${ }^{31}$ A separate analysis of the OSAKA study $(\mathrm{N}=96)$ indicated tear mucin concentration may be lower in those with high daily digital screen use. ${ }^{42}$ Mean mucin 5AC concentration was lower in those using digital screens $>7$ hours per day compared to $<5$ hours per day, providing a potential underlying reason why the long duration of digital screen use results in a more rapid tear breakup time. ${ }^{42}$ These studies support a link between digital screen use and specific changes in the composition of the tear film.

When individuals already diagnosed with DED were examined, greater digital screen use was associated with several ocular surface metrics. Wu et al found that individuals with dry eye who had worked at digital screens for a long time (mean of 8.3 hours daily for a mean of 7.9 years; 106 eyes of 53 patients) had shorter tear breakup time, higher corneal fluorescein staining, and greater OSDI scores than individuals with dry eye who had worked at digital screens for a shorter time (mean of 3.2 hours daily for 5.3 years; 80 eyes of 40 patients). ${ }^{43}$ Schirmer I scores were in the normal range for both groups. The long-time screen workers were also found to have higher lid margin abnormality scores, higher meiboscores (ie, greater meibomian gland loss), and worse expression of meibum than the short-time screen workers. ${ }^{43}$ These findings suggest individuals with dry eye and long durations of digital screen use may suffer greater dry eye severity as evidenced by reduced tear breakup time, ocular surface staining, and meibomian gland dysfunction signs.

\section{Effect of Digital Screen Use on Blinking Dynamics}

The most prevalent hypothesis to explain the link between digital screen use and dry eye is digital screen use influences blinking dynamics by reducing both blink rate and blink completeness, leading to increased ocular surface dryness. $^{31,44-47}$ Aqueous tears evaporate from the tear film during the interval between each blink, and full blinking is required to replenish the tear film by distributing tears (from lacrimal glands) and lipids (from meibomian glands) over the ocular surface. Thus, reduced and incomplete blinking results in ocular surface dryness because it allows for greater evaporative loss, which could, over time, potentially initiate the DED cycle. ${ }^{48}$ Interestingly, individuals who have dry eye typically blink more frequently than individuals without dry eye, which may be an attempt to compensate for tear film instability. ${ }^{45}$

The hypothesis that digital screens lead to dry eye by affecting blinking is supported by several studies showing that blinking dynamics are altered when participants use digital screens. ${ }^{37,49-51}$ Blink rates during reading tasks on digital devices have been found to decrease compared to rest conditions. ${ }^{46,50,52}$ An early study by Tsubota and Nakamori reported the mean rate of blinking in 104 office workers was 22 blinks per minute for a relaxed condition, 10 per minute while reading a book at a table, and 
7 per minute while viewing text on a video screen. ${ }^{46}$ Similarly, blink rate has been found to decrease compared to rest conditions when participants have done active tasks on a computer such as playing computer or video games. ${ }^{37,49}$ Acosta et al found that blink rate was reduced to about $42 \%$ of the control when participants played a computer game (ie, Solitaire) compared to a rest condition. Blink rate subsequently returned to rest levels when participants ceased playing the computer game. ${ }^{49}$ Blink rate has also been found to decrease during an active computer task (arranging words in alphabetical order) compared to the blink rate found when participants were engaged in relaxed conversation. ${ }^{51}$ Notably, blink rate tends to be higher during conversation than when at rest, making conversational conditions less than an ideal comparison condition. ${ }^{53}$ In addition to decreases in blink rate, the percentage of incomplete blinks increases with active digital screen tasks. Cardona et al found the percentage of incomplete blinks increased compared to baseline during video-game playing. ${ }^{37}$ Specifically, $80 \%$ of blinks were incomplete in the baseline condition, whereas $92 \%$ and $88 \%$ of blinks were incomplete during video-game playing. ${ }^{37}$ These data also point to the hypothesis that digital screen use may result in dry eye via its effect on blinking dynamics.

Interestingly, while reading on a digital screen has been found to decrease blink rate, reading hard-copy material also decreases blink rate. ${ }^{50,52}$ Indeed, some studies have found blink rate during hard-copy reading is similar to or even lower than blink rate while reading from a digital screen. ${ }^{50,52,54}$ Abusharha found blink rate decreased from a mean of 19.74 per minute at baseline to 14.93 per minute when reading on an electronic tablet and 11.35 per minute when reading the same text from a book at the same distance as the tablet. ${ }^{52}$ In other studies, blink rates were comparable between digital-screen and hard-copy reading conditions. $^{50,55}$ This is consistent with the finding that ocular symptoms such as blurred vision, burning symptoms, and tearing were increased following reading from an e-book device or a printed book; however, burning symptoms were significantly higher following e-book reading. ${ }^{56}$ Notably, the percentage of incomplete blinks has been found to be higher in the digital-screen versus the hard-copy reading conditions. ${ }^{55}$ For example, Chu et al found the percentage of incomplete blinks was $7.02 \%$ in a computer reading condition compared to $4.33 \%$ in a hard-copy reading condition. ${ }^{55}$ These results emphasize that reading on a digital screen may promote adverse effects by reducing both blink rate and blink completeness.

Findings have suggested that the negative effect of digital screen use on blinking dynamics depends on the cognitive demands of the task. ${ }^{57,58}$ Blink rate is decreased when participants are engaged in an active computer task compared to a passive task. ${ }^{57,58}$ Skotte et al found mean blink frequency was 5.0 per minute when connecting a sequence of small dots with the computer mouse and 16.0 per minute when passively watching a video. ${ }^{58}$ Similarly, blink rate decreased when participants were engaged in a computer game with a high rate of presentation of visual information (a fast-paced 3D shooter game) compared to a low rate of presentation of visual information (a slow-paced 2D strategy game). ${ }^{37}$ These results suggest individuals who are actively engaged on digital screens may be at risk of inadequate blinking, which could potentially lead to more dry eye symptoms. It is worth noting that further research is needed to address limitations in current understanding of blink rates and how to best assess them. ${ }^{59-61}$

\section{Impact of Dry Eye Disease}

The relationship between screen use and dry eye is especially significant because dry eye may have a negative impact on quality of life, possibly due to ocular pain and visual disturbances that can result from tear film instability. ${ }^{2}$ In a large study $(\mathrm{N}=3275)$, the relationship between dry eye and quality of life was shown for both health-related quality of life and vision-related quality of life. ${ }^{6}$ The largest difference in vision-related quality of life between those with and without dry eye symptoms occurred in the ocular pain subscale; however, several subscales showed a significant difference between groups, including distance-vision activities, near-vision activities, and vision-related role limitations, suggesting that dry eye symptoms may interfere with daily functioning. Furthermore, symptoms of depression were 64\% more likely to be reported in those with dry eye symptoms than in those without dry eye symptoms. ${ }^{6}$ Importantly, there is an association between dry eye and mental health among individuals with high use of digital screens. Tounaka et al found that for 163 university employees who used digital screens for work, quality of life as measured by the 36-Item Short Form Health Survey (SF-36) was significantly lower for males with dry eye than for males without dry eye. ${ }^{62}$ This difference was driven by the mental component of the SF-36. ${ }^{62}$ 
In addition to affecting quality of life, dry eye contributes a substantial economic burden. Direct medical costs for DED account for an estimated $\$ 3.84$ billion (2008 US dollars) burden on the United States healthcare system. ${ }^{63,64}$ Indirect costs related to reduced work productivity account for an even larger burden to society, at $\$ 55.4$ billion (2008 US dollars). ${ }^{63,64}$ Studies have examined the relationship between DED and work productivity in digital screen users. In a large study of office workers $(\mathrm{N}=553)$, work limitations were greater for individuals with a diagnosis of definite DED than for those without a diagnosis of DED. ${ }^{10}$ The estimated loss of productivity amounted to 3.1 working days per year for the individuals with definite DED. Subscale analysis showed limitations in time management and limitations in mental and interpersonal functioning were greater in those with definite DED. ${ }^{10}$ Similar findings were shown in a survey of office workers with a high level of reading or computer work. Nearly $70 \%$ of respondents $(\mathrm{N}=505)$ indicated symptoms of dry eye inhibited some work activities, and over 5\% indicated symptoms of dry eye inhibited work activities most of the time. ${ }^{65}$ Furthermore, symptoms such as stinging, burning, itching, irritation, photophobia, blurry vision, and eye pain were reported to occur more frequently at work than at home. ${ }^{65}$ Therefore, early diagnosis and management of dry eye is important for preventing and reducing the possible negative impact of DED on quality of life and work productivity.

\section{Prevention, Diagnosis, and Management of Dry Eye Prevention of Digital Screen-Induced Dry Eye}

Strategies to prevent or mitigate dry eye in digital screen users may involve behavioral modifications such as blinking exercises or periodically resting the eyes as well as environmental modifications. ${ }^{45,66}$ There is some evidence to support that blinking or resting the eyes may be effective at reducing symptoms of dry eye. Kim et al investigated the effect of blinking exercises in 41 participants with symptoms of dry eye. ${ }^{45}$ The blinking exercises consisted of closing the eyes normally for 2 seconds, closing the eyes normally again for 2 seconds, and then squeezing the eyelids together tightly for 2 seconds. After 28 days of the blinking exercises, tear meniscus height and lipid layer thickness did not show a change; however, symptoms of dry eye (OSDI and Dry Eye Questionnaire - 5
Item [DEQ-5] score) decreased, lipid layer quality grading increased, and tear breakup time increased. Furthermore, blink rate (which is normally increased in patients with DED) decreased as did the percentage of incomplete blinks, even within 5 minutes of performing 20 cycles of the exercises in the clinic, suggesting a benefit of the blinking exercises. ${ }^{45} \mathrm{~A}$ different strategy for preventing dry eye from digital screens, termed "blind working," has also been evaluated ${ }^{66}$ Blind working involves closing the eyes when vision is not required. A study of 10 office workers with normal eyes found that individuals implemented "blind working" on average 7.4 times over a 20minute period with a mean duration of 8.6 seconds per event when encouraged to do so. Compared to normal working, visual analog scale scores for dry eye, ocular fatigue, and blurred vision were reduced in the blind working condition. ${ }^{66}$ While further research is needed to verify these strategies as ways to prevent DED, they present potential ways to counteract the negative effects of digital screen use on the eyes. A third possible strategy for preventing dry eye is the recommended 20-20-20 rule to help prevent digital eye strain symptoms. It states that for every 20 minutes of screen use, patients should take a 20 -second break and look at something 20 feet away. ${ }^{67}$ The effectiveness of the 20-20-20 rule for patients with dry eye has not yet been investigated; however, this strategy may potentially be helpful for preventing dry eye by allowing time for natural blinking, which replenishes the tear film.

Environmental modifications may also be considered to prevent or mitigate dry eye in digital screen users. A randomized crossover study of 44 computer users found that 1 hour of computer use with a desktop humidifier was associated with an improvement in tear breakup time and subjective comfort compared with 1 hour of computer use without the humidifier. ${ }^{68}$ No significant difference was observed in lipid-layer grade or tear meniscus height for either condition. ${ }^{6}$ These results suggest that desktop humidifier use may provide relief for symptoms of dry eye and help to prevent DED in digital screen users by reducing tear evaporation. However, the preventative benefits of humidifiers for DED require further investigation. The effect of virtual reality headsets on the ocular surface has also been investigated. ${ }^{69,70}$ A study of 20 individuals found that 40 minutes of virtual reality headset wear was associated with improvements in tear film lipid grade and tear breakup time compared to conventional computer use. ${ }^{69}$ The virtual reality headset also resulted 
in local increase in temperature and reduced humidity, suggesting that the increase in temperature may have contributed to the improvements in lipid grade and tear break up time. ${ }^{69}$ Similarly, in a study of 12 individuals, increased lipid layer thickness was found with a virtual reality headset compared to conventional computer use. ${ }^{70}$ Improvement in the lipid layer is relevant for the prevention of dry eye, as it can improve tear film stability and reduce tear evaporation. ${ }^{69}$ The results of these studies indicate there are potential behavioral and environmental modifications that may help with the prevention of DED. Importantly, patients are likely to only implement such strategies if they understand the relationship between dry eye and digital screen use and are aware of such strategies. Therefore, it is essential that clinicians educate patients on the potential negative effects of digital screen use on the ocular surface and possible preventative strategies, especially with patients who are at risk and who already report dry eye symptoms.

\section{Diagnosis and Management of Dry Eye Disease}

For patients with reported dry eye symptoms and observed clinical signs, accurate diagnosis of DED is important for guiding effective management of DED. Any individual who is at risk of DED can be screened for DED with the DEQ-5, OSDI, or Standard Patient Evaluation of Eye Dryness (SPEED) surveys, which can indicate if that individual might have DED. ${ }^{71}$ Subsequently, clinical tests for DED can be performed, which include tear breakup time, ocular surface staining, tear meniscus height, and direct evaluation of the eyelids, eyelashes, and meibomian gland secretions. Laboratory/imaging tests for dry eye include tear film osmolarity, the presence of inflammatory markers, lipid layer analysis, and visualization of the meibomian glands. It is important that other conditions that mimic DED are ruled out and possible comorbidities are investigated so the most appropriate treatments can be prescribed. Clinicians may encounter patients who have DED signs without symptoms or symptoms without signs. ${ }^{1}$ In these cases, clinicians may educate patients on healthy digital screen habits to prevent progression of the condition. If a diagnosis of DED is made, evaluation of severity and the extent to which the DED manifests as evaporative dry eye or aqueous deficient dry eye is important for further guiding treatment decisions. ${ }^{71}$
Treatment of DED aims to restore homeostasis to the tear film and to provide options to prevent the return of DED. ${ }^{12}$ A variety of treatments are available for DED including treatments to address tear film insufficiency, meibomian gland dysfunction, and inflammation, the choice of which depends on the characteristics, contributing factors, and severity of the DED. ${ }^{12}$ A number of clinical guidelines for the management of DED have been developed. These include the Tear Film and Ocular Surface Society Dry Eye Workshop II (TFOS DEWS II) staged management and treatment recommendations for DED, ${ }^{12}$ the American Society of Cataract and Refractive Surgery (ASCRS) preoperative ocular surface disease algorithm, ${ }^{72}$ and the recommended treatment options of the Cornea, External Disease, and Refractive Society (CEDARS) group. ${ }^{73}$

For DED that may be related to digital screen use, a combination of ocular therapies and lifestyle modifications may prove beneficial. An important part of patient education includes discussion on use of a preservative-free tear supplement and practice of healthier digital habits and screen engagement methods. Deliberate full blinking exercises, frequent breaks, and generally reduced digital device use are some potentially helpful healthy digital habits. In addition, strategies that may help in management of computer vision syndrome - a broader category of eye and vision problems related to digital screen use-such as enlarging font size, reducing glare, improving contrast, and using a downward gaze may also be beneficial for those with symptoms of dry eye. ${ }^{39,74-76}$ As eye strain from digital screens may make overall symptoms worse, diagnosis and management of refractive error and/or binocular dysfunction may help to alleviate symptoms associated with digital screen use. ${ }^{74,75}$ However, given that it may be difficult for some patients to reduce high use of digital screens for work and communication in the modern era, there may be a need for regular follow-up and proactive management strategies that include ocular therapies in addition to digital habits. Because there is limited knowledge about the efficacy of different treatment strategies in individuals with DED and high use of digital screens, future research may elucidate the best long-term management strategies for these patients.

\section{Conclusions}

Digital screen use is part of everyday life and is a risk factor for DED. A likely explanation for the relationship between digital screen use and DED is that reduced blink 
rate and increased percentage of incomplete blinks during digital screen use can lead to ocular surface dryness, which may facilitate the development of DED when individuals are actively engaged on digital screens for long periods of time. Therefore, prevention of DED may involve deliberately blinking the eyes and allowing the eyes time to blink naturally, as well as environmental modifications aimed at reducing tear evaporation. Eye care professionals should inquire about patients' digital habits and dry eye symptoms during annual eye exams. This will help to identify those at risk of DED and those who would benefit from a screening for DED or evaluation for clinical signs of DED. It is important for eye care professionals to raise patients' awareness of the link between DED and digital screen use and of possible prevention strategies. For patients who exhibit symptoms, accurate DED diagnosis by an eye care professional is important for determining appropriate treatment. For patients with DED, education on lifestyle modifications and comprehensive ocular therapies should be considered. Further research is needed to inform on optimal management strategies for DED in individuals with high use of digital screens.

\section{Acknowledgments}

Medical writing support was provided by IMPRINT Science, New York, NY, and was funded by Novartis Pharmaceuticals Corporation. This manuscript was developed in accordance with Good Publication Practice (GPP3) guidelines. Authors had full control of the content and made the final decision on all aspects of this publication.

\section{Funding}

This work and editorial support was funded by Novartis Pharmaceuticals Corporation, East Hanover, NJ, USA.

\section{Disclosure}

Zaina Al-Mohtaseb is a consultant to Alcon, Zeiss, Novartis, and Bausch \& Lomb. Scott Schachter is Director, US Medical Affairs, Presbyopia and Ocular Surface Disease, Allergan, an AbbVie company. Bridgitte Shen Lee reports relationships with Alcon, CooperVision, Essilor, Johnson \& Johnson Vision, Kala Pharmaceuticals, Lumenis Inc, SightGlass, and Sun Pharmaceutical Industries Ltd. Jaclyn Garlich reports relationships with Allergan, Dompé, Novartis, Orasis, Quidel Corporation, Tarsus Pharmaceuticals, and Zeiss. William Trattler reports relationships with Alcon, Allergan, Azura Ophthalmics, Bausch
Health, Dompé, Glaukos, Johnson \& Johnson, Kala Pharmaceuticals, NovaBay Pharmaceuticals, Novartis, Sight Sciences, and Sun Pharmaceutical Industries Ltd. He is also an investor for EyeSafe. The authors report no other conflicts of interest in this work.

\section{References}

1. Craig JP, Nichols KK, Akpek EK, et al. TFOS DEWS II definition and classification report. Ocul Surf. 2017;15(3):276-283. doi:10.1016/j.jtos.2017.05.008

2. Stapleton F, Alves M, Bunya VY, et al. TFOS DEWS II epidemiology report. Ocul Surf. 2017;15(3):334-365.

3. Clayton JA. Dry eye. $N$ Engl J Med. 2018;379(11):e19.

4. Kawashima M, Yamatsuji M, Yokoi N, et al. Screening of dry eye disease in visual display terminal workers during occupational health examinations: the Moriguchi Study. J Occup Health. 2015;57 (3):253-258. doi:10.1539/joh.14-0243-OA

5. Ahn JM, Lee SH, Rim TH, et al. Prevalence of and risk factors associated with dry eye: the Korea National Health and Nutrition Examination Survey 2010-2011. Am J Ophthalmol. 2014;158 (6):1205-1214e.7. doi:10.1016/j.ajo.2014.08.021

6. Paulsen AJ, Cruickshanks KJ, Fischer ME, et al. Dry eye in the Beaver Dam Offspring Study: prevalence, risk factors, and health-related quality of life. Am $J$ Ophthalmol. 2014;157 (4):799-806. doi:10.1016/j.ajo.2013.12.023

7. Karakus S, Mathews PM, Agrawal D, Henrich C, Ramulu PY, Akpek EK. Impact of dry eye on prolonged reading. Optom Vis Sci. 2018;95(12):1105-1113. doi:10.1097/OPX.0000000000001303

8. Mathews PM, Ramulu PY, Swenor BS, Utine CA, Rubin GS, Akpek EK. Functional impairment of reading in patients with dry eye. $\mathrm{Br}$ J Ophthalmol. 2017;101(4):481-486. doi:10.1136/bjophthalmol-2015308237

9. Nichols KK, Bacharach J, Holland E, et al. Impact of dry eye disease on work productivity, and patients' satisfaction with over-the-counter dry eye treatments. Invest Ophthalmol Vis Sci. 2016;57 (7):2975-2982. doi:10.1167/iovs.16-19419

10. Uchino M, Uchino Y, Dogru M, et al. Dry eye disease and work productivity loss in visual display users: the Osaka Study. $\mathrm{Am}$ J Ophthalmol. 2014;157(2):294-300. doi:10.1016/j.ajo.2013.10.014

11. Gomes JAP, Azar DT, Baudouin C, et al. TFOS DEWS II iatrogenic report. Ocul Surf. 2017;15(3):511-538.

12. Jones L, Downie LE, Korb D, et al. TFOS DEWS II management and therapy report. Ocul Surf. 2017;15(3):575-628.

13. LeBlanc AG, Gunnell KE, Prince SA, Saunders TJ, Barnes JD, Chaput J-P. The ubiquity of the screen: an overview of the risks and benefits of screen time in our modern world. Transl $\mathrm{J} \mathrm{Am}$ Sports Med. 2017;2(17):104-113.

14. Courtin R, Pereira B, Naughton G, et al. Prevalence of dry eye disease in visual display terminal workers: a systematic review and meta-analysis. BMJ Open. 2016;6(1):e009675. doi:10.1136/bmjopen2015-009675

15. Sheppard AL, Wolffsohn JS. Digital eye strain: prevalence, measurement and amelioration. BMJ Open Ophthalmol. 2018;3(1):e00146. doi:10.1136/bmjophth-2018-000146

16. Vanderloo LM, Carsley S, Aglipay M, Cost KT, Maguire J, Birken CS. Applying harm reduction principles to address screen time in young children amidst the COVID-19 pandemic. J Dev Behav Pediatr. 2020;41(5):335-336. doi:10.1097/DBP.0000000000000825

17. Walsh JJ, Barnes JD, Tremblay MS, Chaput J-P. Associations between duration and type of electronic screen use and cognition in US children. Comput Human Behav. 2020;108:106312. doi:10.1016/ j.chb.2020.106312 
18. Hussaindeen JR, Gopalakrishnan A, Sivaraman V, Swaminathan M. Managing the myopia epidemic and digital eye strain post COVID-19 pandemic - What eye care practitioners need to know and implement? Indian $J$ Ophthalmol. 2020;68(8):1710-1712. doi:10.4103/ijo. IJO_2147_20

19. King DL, Delfabbro PH, Billieux J, Potenza MN. Problematic online gaming and the COVID-19 pandemic. J Behav Addict. 2020;9 (2):184-186. doi:10.1556/2006.2020.00016

20. Majumdar P, Biswas A, Sahu S. COVID-19 pandemic and lockdown: cause of sleep disruption, depression, somatic pain, and increased screen exposure of office workers and students of India. Chronobiol Int. 2020;37(8):1191-1200.

21. Schmidt SCE, Anedda B, Burchartz A, et al. Physical activity and screen time of children and adolescents before and during the COVID-19 lockdown in Germany: a natural experiment. Sci Rep. 2020;10(1):21780. doi:10.1038/s41598-020-78438-4

22. Nagata JM, Abdel Magid HS, Pettee Gabriel K. Screen time for children and adolescents during the coronavirus disease 2019 pandemic. Obesity (Silver Spring). 2020;28(9):1582-1583. doi:10.1002/oby.22917

23. Mukhopadhyay S, Booth AL, Calkins SM, et al. Leveraging technology for remote learning in the era of COVID-19 and social distancing. Arch Pathol Lab Med. 2020;144(9):1027-1036. doi:10.5858/arpa.2020-0201-ED

24. Vollbrecht PJ, Porter-Stransky KA, Lackey-Cornelison WL. Lessons learned while creating an effective emergency remote learning environment for students during the COVID-19 pandemic. Adv Physiol Educ. 2020;44(4):722-725. doi:10.1152/advan.00140.2020

25. Bhattacharya S, Saleem SM, Singh A. Digital eye strain in the era of COVID-19 pandemic: an emerging public health threat. Indian J Ophthalmol. 2020;68(8):1709-1710. doi:10.4103/ijo.IJO_1782_20

26. Rolland B, Haesebaert F, Zante E, Benyamina A, Haesebaert J, Franck N. Global changes and factors of increase in caloric/salty food intake, screen use, and substance use during the early COVID-19 containment phase in the general population in France: survey study. JMIR Public Health Surveill. 2020;6(3):e19630. doi:10.2196/19630

27. Colley RC, Bushnik T, Langlois K. Exercise and screen time during the COVID-19 pandemic. Health Rep. 2020;31(6):3-11.

28. Giannaccare G, Vaccaro S, Mancini A, Scorcia V. Dry eye in the COVID-19 era: how the measures for controlling pandemic might harm ocular surface. Graefes Arch Clin Exp Ophthalmol. 2020;258 (11):2567-2568. doi:10.1007/s00417-020-04808-3

29. Uchino M, Schaumberg DA, Dogru M, et al. Prevalence of dry eye disease among Japanese visual display terminal users. Ophthalmology. 2008;115(11):1982-1988. doi:10.1016/j.ophtha.2008.06.022

30. Inomata T, Iwagami M, Nakamura M, et al. Characteristics and risk factors associated with diagnosed and undiagnosed symptomatic dry eye using a smartphone application. JAMA Ophthalmol. 2020;138 (1):58-68. doi:10.1001/jamaophthalmol.2019.4815

31. Uchino M, Yokoi N, Uchino Y, et al. Prevalence of dry eye disease and its risk factors in visual display terminal users: the Osaka Study. Am J Ophthalmol. 2013;156(4):759-766. doi:10.1016/j.ajo.20 13.05 .040

32. Hanyuda A, Sawada N, Uchino M, et al. Physical inactivity, prolonged sedentary behaviors, and use of visual display terminals as potential risk factors for dry eye disease: JPHC-NEXT study. Ocul Surf. 2020;18(1):56-63. doi:10.1016/j.jtos.2019.09.007

33. Wang MTM, Muntz A, Mamidi B, Wolffsohn JS, Craig JP. Modifiable lifestyle risk factors for dry eye disease. Cont Lens Anterior Eye. 2021;101409. doi:10.1016/j.clae.2021.01.004

34. Wolffsohn JS, Wang MTM, Vidal-Rohr M, et al. Demographic and lifestyle risk factors of dry eye disease subtypes: a cross-sectional study. Ocul Surf. 2021;21:58-63. doi:10.1016/j.jtos.2021.05.001
35. Moon JH, Lee MY, Moon NJ. Association between video display terminal use and dry eye disease in school children. $J$ Pediatr Ophthalmol Strabismus. 2014;51(2):87-92. doi:10.3928/0191391320140128-01

36. Moon JH, Kim KW, Moon NJ. Smartphone use is a risk factor for pediatric dry eye disease according to region and age: a case control study. BMC Ophthalmol. 2016;16(1):188. doi:10.1186/s12886-0160364-4

37. Cardona G, Garcia C, Seres C, Vilaseca M, Gispets J. Blink rate, blink amplitude, and tear film integrity during dynamic visual display terminal tasks. Curr Eye Res. 2011;36(3):190-197. doi:10.3109/ 02713683.2010 .544442

38. Akkaya S, Atakan T, Acikalin B, Aksoy S, Ozkurt Y. Effects of long-term computer use on eye dryness. North Clin Istanb. 2018;5 (4):319-322.

39. Talens-Estarelles C, Sanchis-Jurado V, Esteve-Taboada JJ, Pons AM, Garcia-Lazaro S. How do different digital displays affect the ocular surface? Optom Vis Sci. 2020;97(12):1070-1079. doi:10.1097/ OPX.0000000000001616

40. Nakamura S, Kinoshita S, Yokoi N, et al. Lacrimal hypofunction as a new mechanism of dry eye in visual display terminal users. PLoS One. 2010;5(6):e11119. doi:10.1371/journal.pone.0011119

41. Kojima T, Ibrahim OM, Wakamatsu T, et al. The impact of contact lens wear and visual display terminal work on ocular surface and tear functions in office workers. Am J Ophthalmol. 2011;152(6):933-940. e2. doi:10.1016/j.ajo.2011.05.025

42. Uchino Y, Uchino M, Yokoi N, et al. Alteration of tear mucin 5AC in office workers using visual display terminals: the Osaka Study. JAMA Ophthalmol. 2014;132(8):985-992. doi:10.1001/jamaophthalmol.20 14.1008

43. Wu H, Wang Y, Dong N, et al. Meibomian gland dysfunction determines the severity of the dry eye conditions in visual display terminal workers. PLoS One. 2014;9(8):e105575. doi:10.1371/journal. pone. 0105575

44. Cardona G, Gomez M, Quevedo L, Gispets J. Effects of transient blur and VDT screen luminance changes on eyeblink rate. Cont Lens Anterior Eye. 2014;37(5):363-367. doi:10.1016/j.clae.2014.05.005

45. Kim AD, Muntz A, Lee J, Wang MTM, Craig JP. Therapeutic benefits of blinking exercises in dry eye disease. Cont Lens Anterior Eye. 2020;44(3):101329.

46. Tsubota K, Nakamura S. Dry eyes and video display terminals. $N$ Engl J Med. 1993;328(8):584. doi:10.1056/NEJM19930225 3280817

47. Yokoi N, Uchino M, Uchino Y, et al. Importance of tear film instability in dry eye disease in office workers using visual display terminals: the Osaka study. Am J Ophthalmol. 2015;159(4):748-754. doi:10.1016/j.ajo.2014.12.019

48. Bron AJ, de Paiva CS, Chauhan SK, et al. TFOS DEWS II pathophysiology report. Ocul Surf. 2017;15(3):438-510.

49. Acosta MC, Gallar J, Belmonte C. The influence of eye solutions on blinking and ocular comfort at rest and during work at video display terminals. Exp Eye Res. 1999;68(6):663-669. doi:10.1006/ exer. 1998.0656

50. Argiles M, Cardona G, Perez-Cabre E, Rodriguez M. Blink rate and incomplete blinks in six different controlled hard-copy and electronic reading conditions. Invest Ophthalmol Vis Sci. 2015;56 (11):6679-6685. doi:10.1167/iovs.15-16967

51. Freudenthaler N, Neuf H, Kadner G, Schlote T. Characteristics of spontaneous eyeblink activity during video display terminal use in healthy volunteers. Graefes Arch Clin Exp Ophthalmol. 2003;241 (11):914-920. doi:10.1007/s00417-003-0786-6

52. Abusharha AA. Changes in blink rate and ocular symptoms during different reading tasks. Clin Optom (Auckl). 2017;9:133-138. doi:10.2147/OPTO.S142718 
53. Bentivoglio AR, Bressman SB, Cassetta E, Carretta D, Tonali P, Albanese A. Analysis of blink rate patterns in normal subjects. Mov Disord. 1997;12(6):1028-1034. doi:10.1002/mds.870120629

54. Rosenfield M, Jahan S, Nunez K, Chan K. Cognitive demand, digital screens and blink rate. Comput Human Behav. 2015;51:403-406. doi:10.1016/j.chb.2015.04.073

55. Chu CA, Rosenfield M, Portello JK. Blink patterns: reading from a computer screen versus hard copy. Optom Vis Sci. 2014;91 (3):297-302. doi:10.1097/OPX.0000000000000157

56. Prabhasawat P, Pinitpuwadol W, Angsriprasert D, Chonpimai P, Saiman M. Tear film change and ocular symptoms after reading printed book and electronic book: a crossover study. Jpn J Ophthalmol. 2019;63 (2):137-144. doi:10.1007/s10384-018-00648-1

57. Himebaugh NL, Begley CG, Bradley A, Wilkinson JA. Blinking and tear break-up during four visual tasks. Optom Vis Sci. 2009;86(2): E106-114. doi:10.1097/OPX.0b013e318194e962

58. Skotte JH, Nojgaard JK, Jorgensen LV, Christensen KB, Sjogaard G. Eye blink frequency during different computer tasks quantified by electrooculography. Eur J Appl Physiol. 2007;99(2):113-119. doi:10.1007/s00421-006-0322-6

59. McMonnies CW. The clinical and experimental significance of blinking behavior. $J$ Optom. 2020;13(2):74-80. doi:10.1016/j. optom.2019.09.002

60. Cruz AA, Garcia DM, Pinto CT, Cechetti SP. Spontaneous eyeblink activity. Ocul Surf. 2011;9(1):29-41. doi:10.1016/S1542-0124(11) 70007-6

61. Rodriguez JD, Lane KJ, Ousler GW 3rd, Angjeli E, Smith LM, Abelson MB. Blink: characteristics, controls, and relation to dry eyes. Curr Eye Res. 2018;43(1):52-66. doi:10.1080/ 02713683.2017.1381270

62. Tounaka K, Yuki K, Kouyama K, et al. Dry eye disease is associated with deterioration of mental health in male Japanese university staff. Tohoku J Exp Med. 2014;233(3):215-220. doi:10.1620/tjem.233.215

63. McDonald M, Patel DA, Keith MS, Snedecor SJ. Economic and humanistic burden of dry eye disease in Europe, North America, and Asia: a systematic literature review. Ocul Surf. 2016;14 (2):144-167. doi:10.1016/j.jtos.2015.11.002

64. Yu J, Asche CV, Fairchild CJ. The economic burden of dry eye disease in the United States: a decision tree analysis. Cornea. 2011;30(4):379-387. doi:10.1097/ICO.0b013e3181f7f363
65. van Tilborg MM, Murphy PJ, Evans KS. Impact of dry eye symptoms and daily activities in a modern office. Optom Vis Sci. 2017;94 (6):688-693. doi:10.1097/OPX.0000000000001086

66. Fujita H, Sano K, Baba T, Tanaka T, Ohno-Matsui K. Blind working time in visual display terminal users. J Occup Health. 2019;61 (2):175-181. doi:10.1002/1348-9585.12027

67. American Optometric Association. Computer vision syndrome; 2020. Available from: https://www.aoa.org/healthyeyes/eye-and-visionconditions/computer-vision-syndrome. Accessed November 13, 2020.

68. Wang MTM, Chan E, Ea L, et al. Randomized trial of desktop humidifier for dry eye relief in computer users. Optom Vis Sci. 2017;94(11):1052-1057. doi:10.1097/OPX.0000000000001136

69. Turnbull PRK, Wong J, Feng J, Wang MTM, Craig JP. Effect of virtual reality headset wear on the tear film: a randomised crossover study. Cont Lens Anterior Eye. 2019;42(6):640-645. doi:10.1016/j. clae.2019.08.003

70. Marshev V, Bolloc'h J, Pallamin N, Cochener B, Nourrit V. Impact of virtual reality headset use on eye blinking and lipid layer thickness. J Fr Ophtalmol. 2021. doi:10.1016/j.jfo.2020.09.032

71. Wolffsohn JS, Arita R, Chalmers R, et al. TFOS DEWS II diagnostic methodology report. Ocul Surf. 2017;15(3):539-574.

72. Starr CE, Gupta PK, Farid M, et al. An algorithm for the preoperative diagnosis and treatment of ocular surface disorders. J Cataract Refract Surg. 2019;45(5):669-684. doi:10.1016/j.jcrs.2019.03.023

73. Milner MS, Beckman KA, Luchs JI, et al. Dysfunctional tear syndrome: dry eye disease and associated tear film disorders - new strategies for diagnosis and treatment. Curr Opin Ophthalmol. 2017;27(Suppl 1):3-47.

74. Gowrisankaran S, Sheedy JE. Computer vision syndrome: a review. Work. 2015;52(2):303-314. doi:10.3233/WOR-152162

75. Kaiti R, Shah P, Bogati B, Shyangbo R, Dahal M, Hamal B. Computer vision syndrome: is it being diagnosed and managed properly? Acta Sci Ophthalmol. 2020;3(7):13-20.

76. Lin CW, Yeh FM, Wu BW, Yang CH. The effects of reflected glare and visual field lighting on computer vision syndrome. Clin Exp Optom. 2019;102(5):513-520. doi:10.1111/cxo.12878

77. Hikichi T, Yoshida A, Fukui Y, et al. Prevalence of dry eye in Japanese eye centers. Graefes Arch Clin Exp Ophthalmol. 1995;233 (9):555-558. doi:10.1007/BF00404705
Clinical Ophthalmology

\section{Publish your work in this journal}

Clinical Ophthalmology is an international, peer-reviewed journal covering all subspecialties within ophthalmology. Key topics include: Optometry; Visual science; Pharmacology and drug therapy in eye diseases; Basic Sciences; Primary and Secondary eye care; Patient Safety and Quality of Care Improvements. This journal is indexed on PubMed

\section{Dovepress}

Central and CAS, and is the official journal of The Society of Clinical Ophthalmology (SCO). The manuscript management system is completely online and includes a very quick and fair peer-review system, which is all easy to use. Visit http://www.dovepress.com/ testimonials.php to read real quotes from published authors. 